\title{
PLANIFICACION FAMILIAR EN EL ICSS - VALLE
}

\author{
Dr. Saulo Muñoz D.* \\ Dr. Jorge Solanilla** \\ Dr. Tito Reyes ***
}

Es objetivo de este trabajo presentar los programas y resultados de la Planificación Familiar en el ICSS Valle, pero antes es conveniente revisar someramente las políticas demográficas a través de los tiempos, para situar dentro de este panorama la orientación que seguimos en las conductas propuestas. Si ouservamos desprevenidamente el resumen de conceptos en políticas demográficas, encontramos desde las más optimis tas e ingenuas, hasta las más pesimistas, rayanas en cuadros catastróficos y apocalípticos.

\section{CUADRO N: 1}

\section{POLITICAS DEMOGRAFICAS}

Mandato Bíblico: "Creced y Multiplicaos".

Factores Religiosos.

"Marx": Desarrollo económico y cambio político-sociales.

Igualdad de recursos económicos. Leyes proteccionistas.

Director Instituto Hudson de Futu. rología N. Y.: Crear condiciones técnicas y económicas. Posibilidad de existencia en la tierra.

"Envejecimiento demográfico".
Sociedad consumista.

"Juan XXIII": Problema de concien cia de los cónyuges.

\section{"SEGUISMO DEMOGRAFICO" \\ "O=2.2".}

"Modelo de Sauvy": Huir del envejecimiento. Subordinación económica de la población y búsqueda óptima demográfica.

Leyes Malthusianas.

Planificación Familiar.

Club de Roma año 2000: No comida, no agua, no aire.

Bomba de la Población "Selich". "Fosas de Hambre". Intervención del Estado.

Presidente Johnson 1965: Mayor rentabilidad de las inversiones demográficas frente a las económicas.

Esterilización quirúrgica.

Aborto legalizado.

Métodos coercitivos Dumont-Ma chale.

* .Tefe Dpto. de Obst. y Ginec. ICSS - Valle.

** Director Comité de Perinotología. ICSS-Valle

*:** Director de Profamilia - Cali Gráficas colaboración de la sección de Dibujo. Dpto. de Medicina Preventiva ICSS - Valle. 
Cuando se habla de políticas demográficas se entiende por tal aquellas que buscan la reducción o estabilización de la población con el fin de obtener un desarrollo óptimo de acuerdo a los recursos del país. Pero hablar de desarrollo en sí es muy complejo, pues para unos implica una situación de orden económico en aumento de los capitales y la renta bruta percapita, lo cual sería un concepto muy simplista y materialista. Es importante que se piense también en función de salud en todo su contenido y en cambios institucionales en cuanto a actitudes y tendencias, io cual sí representaría un resultado más integral de desarrollo como resultado de las políticas demográficas.
Presentamos una esquematización del desarrollo como producto de la técnica y de la educación y que está en relación directa con los recursos y sus implicaciones y en relación inversa a la población, considerando las variantes de estas y los grados en la evolución demográfica.

De acuerdo a los cálculos de la población de Colombia en los 15 a 20 años siguientes la habremos duplicado, realidad que no podemos desconocer y por lo tanto debemos afrontar positivamente, presentando programas y creando una conciencia nacional en la búsque. da de soluciones adecuadas a nuestro medio.

\section{COLOMBIA}

\section{GRAFICA № 1}

\section{DISTRIBUCION DEL CRECIMIENTO DE LA POBLACION} EN EL PRESENTE SIGLO-OCHO FECHAS CENSALES

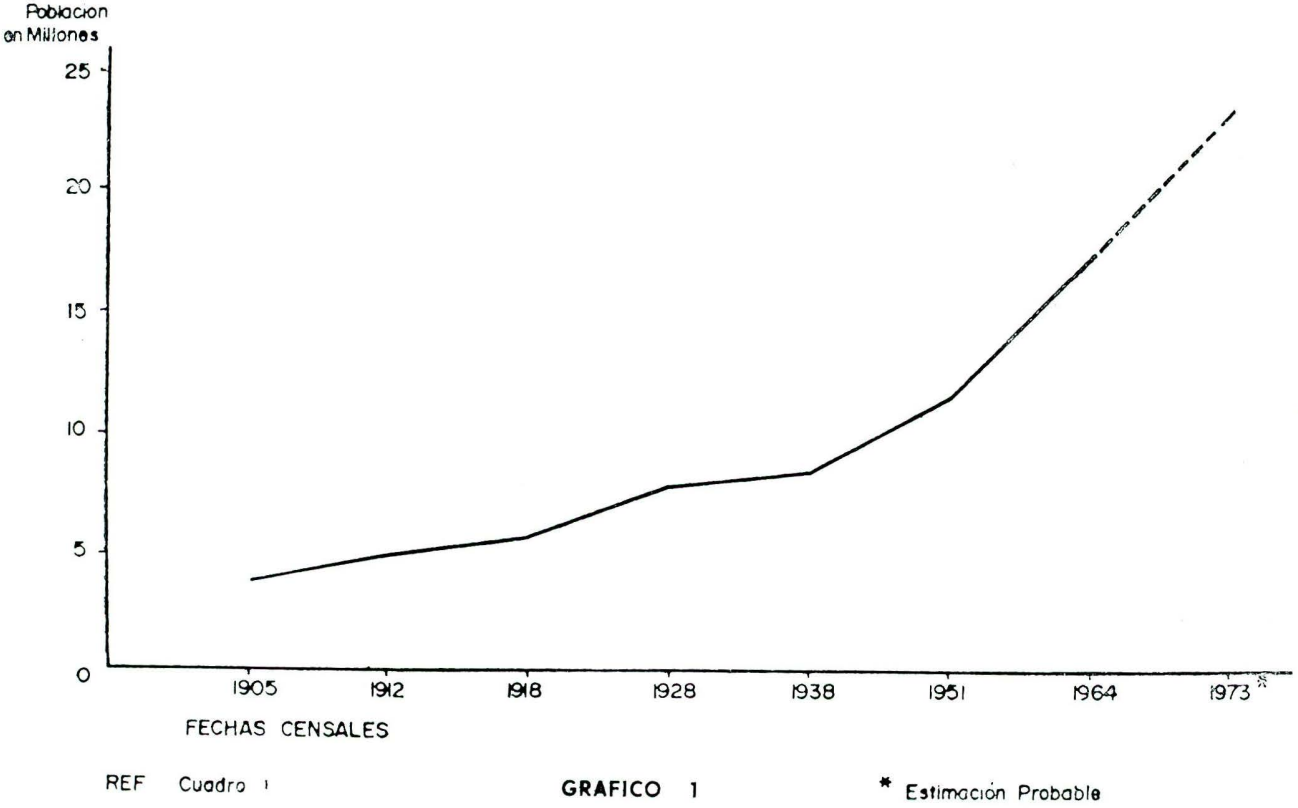


CUADRO N: 2

\section{ESQUEMA SOBRE DESARROLLO EN RELACION CON LOS RECURSOS Y LA POBLACION}

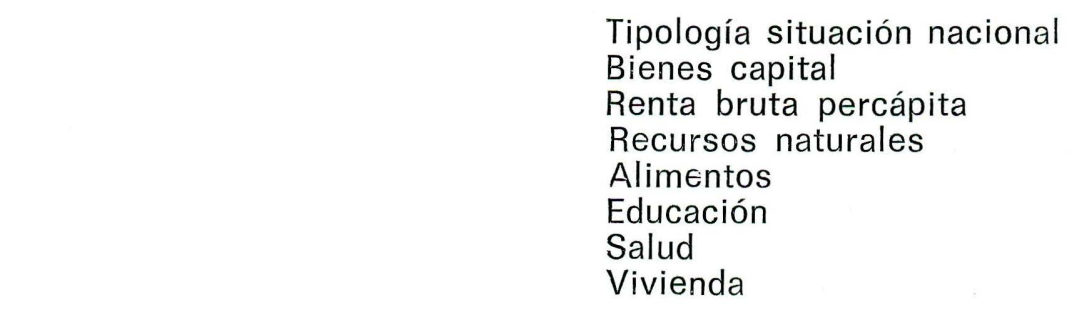

Resultado de la Técnica y Educación

ECONOMICO:

Renta bruta per capita

Bienes capital

Crecimiento recurso a

lgualdad para la población

Industrialización

Planificación

Urbanismo

SALUD:

Calidad

Baja mortalidad

Control polución y

contaminación

Bienestar emocional

Seguridad

Vivienda

CAMBIOS INSTITUCIONALES

Actitudes y tendencias
Tipología situación nacional

Bienes capital

Renta bruta percápita

Recursos naturales

Alimentos

Educación

Salud

Vivienda 


\section{GRAFICA N: 2}

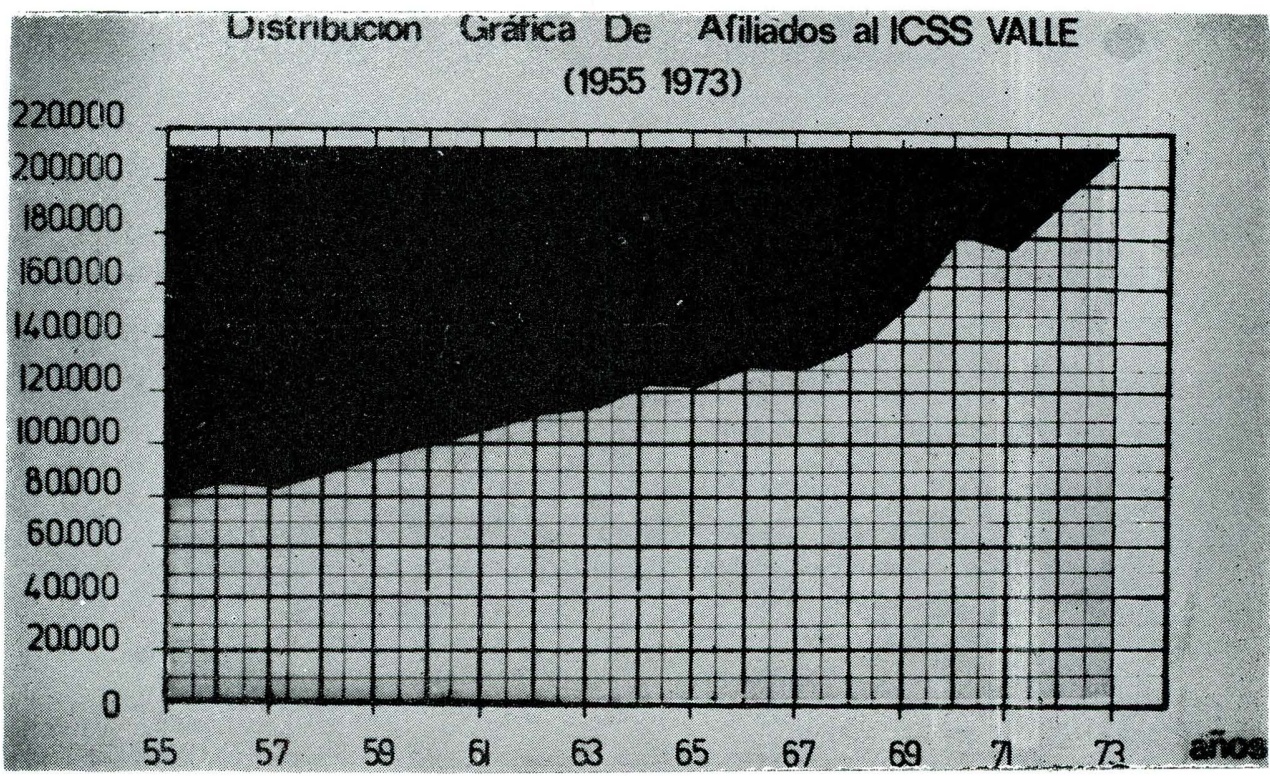

Si tenemos en cuenta el carácter obligatorio de la ley del ICSS, por la cual los trabajadores deben afiliarse y considerando el aumento de la población colombiana así como del sector laboral, el número de usuarios según los programas del gobierno, serían aproximadamente de 3.000 .000 en los próximos años.

La ley presta un servicio médico completo a sus "afiliadas" con derecho a 8 semanas (56 días) de incapacidad por maternidad.

Además a las esposas o compañeras del trabajador Ilamadas "beneficiarias" les da la atención prenatal, parto y cuidado del recién nacido.

Con el actual número de afiliadas y beneficiarias, si los índices de natalidad hubieran seguido creciendo al ritmo que traían hasta antes de las campañas de Planifi- cación, los recursos económicos, médicos y locativos serían inadecuados, y más aún si se considera la magnitud del crecimiento propuesto.

En los Seguros Sociales oficialmente no existe una política demográfica definida y en algunas seccionales apenas se inician programas de planificación.

\section{Material y Métodos}

En Cali desde el año 1969 se estableció una oficina de Profamilia dentro de la Unidad Asistencial Rafael Uribe Uribe que funciona con una promotora, una auxiliar de enfermería y varios médicos. Se sigue un programa de aplicación de dispositivos intra-uterinos post-parto y se las motiva e instruye para que ocupen los ser vicios de Profamilia por fuera de la Institución. 
En la actualidad como método de planificación se utiliza de los 15 a los 20 años programas educacio nales. De los 20 a los 30 regulación por los métodos conocidos y de los 30 en adelante esterilización. Esta se practica de varias maneras y solamente por graves factores socio-económicos en pacientes que tengan más de 30 años y 4 o más hijos o por factores de orden médico los cuales son determinados por una Junta quienes autorizan esta clase de intervenciones.

\section{POLITICA DE POBLACION DEL ICSS EN EL VALLE}

\section{CICLO SOCIO-BIOLOGICO}

$15-$

EDUCACION

$20-$

25 - REGULACION

$30-$

35 - ESTERILIZACION

$40-$

Se estudia la población de afiliados al ICSS en el Valle desde 1955 a 1973 y se discrimina el número de mujeres afiliadas. (GRAFICA $N^{\circ}: 3$ ).

Sobre el total de afiliados se discrimina el número de mujeres afiliadas y beneficiarias (GRAFICA N. 4).

En un cuadro comparativo sobre la mayor causa de hospitalización en el Valle se nota que aproximadamente el $40 \%$ corresponde a pacientes obstétricas. (GRAFICA $\mathrm{N}$ ? $5)$.

Se sacan las tasas de embarazos por 1000 entre afiliadas y beneficiarias del Valle del año 1967 a 1974, encontrando que desde el

\section{A) Factores socio-económicos 30 o más años y 40 más hijos \\ B) Factores médicos}

año 1969 que se organizó Profamilia ha habido una definida disminución en las tasas de nacimientos de las beneficiarias (CUADRO №3 y GRAFICA № 6).

Se estudian los partos de 1956 a 1974 en la Unidad Asistencial Rafael Uribe Uribe. (GRAFICA № 7) y se las discrimina entre afiliadas y beneficiarias (GRAFICA № 8).

Se muestra la forma de terminación de los partos, con un porcentaje de cesáreas las cuales se han incrementado en los últimos años (GRAFICA № 9).

Se presenta el número de abortos tanto en afiliadas como en beneficiarias desde el año 1956 a 1974 (GRAFICA №10). 
GRAFICA N: 3

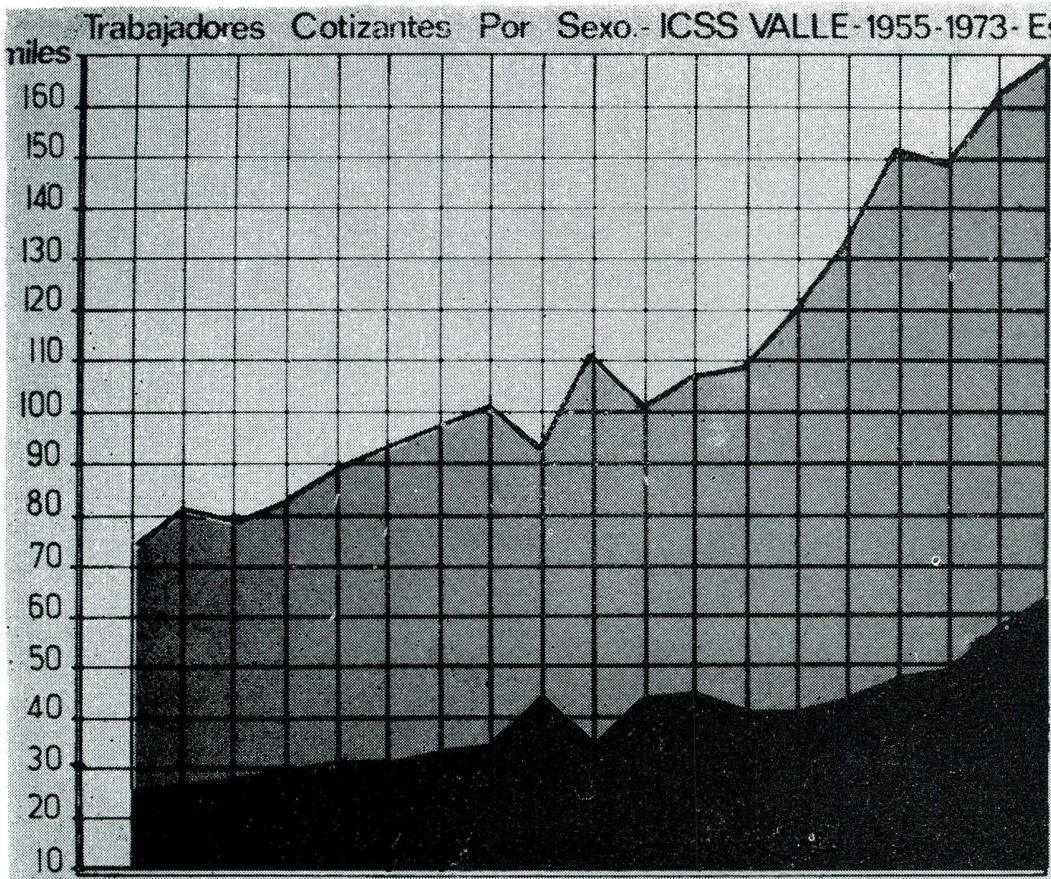

Hombres -

Mujeres -

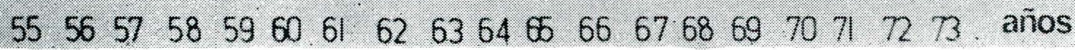

GRAFICA N: 4

7 DISTRIBUCION DE AFILIADOS Y MUJERES:AFILIADAS BENEFICIARIAS

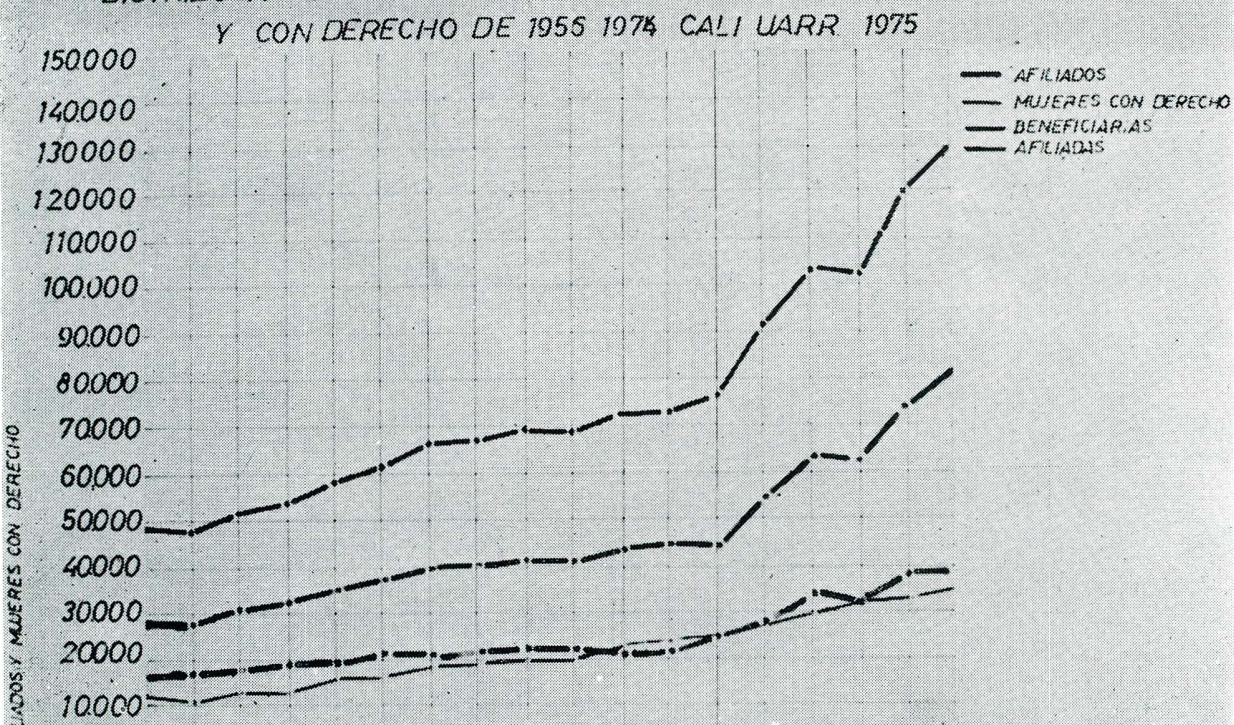

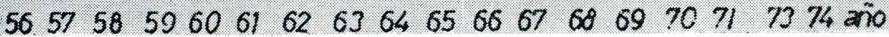


GRAFICA Nㅇ 5

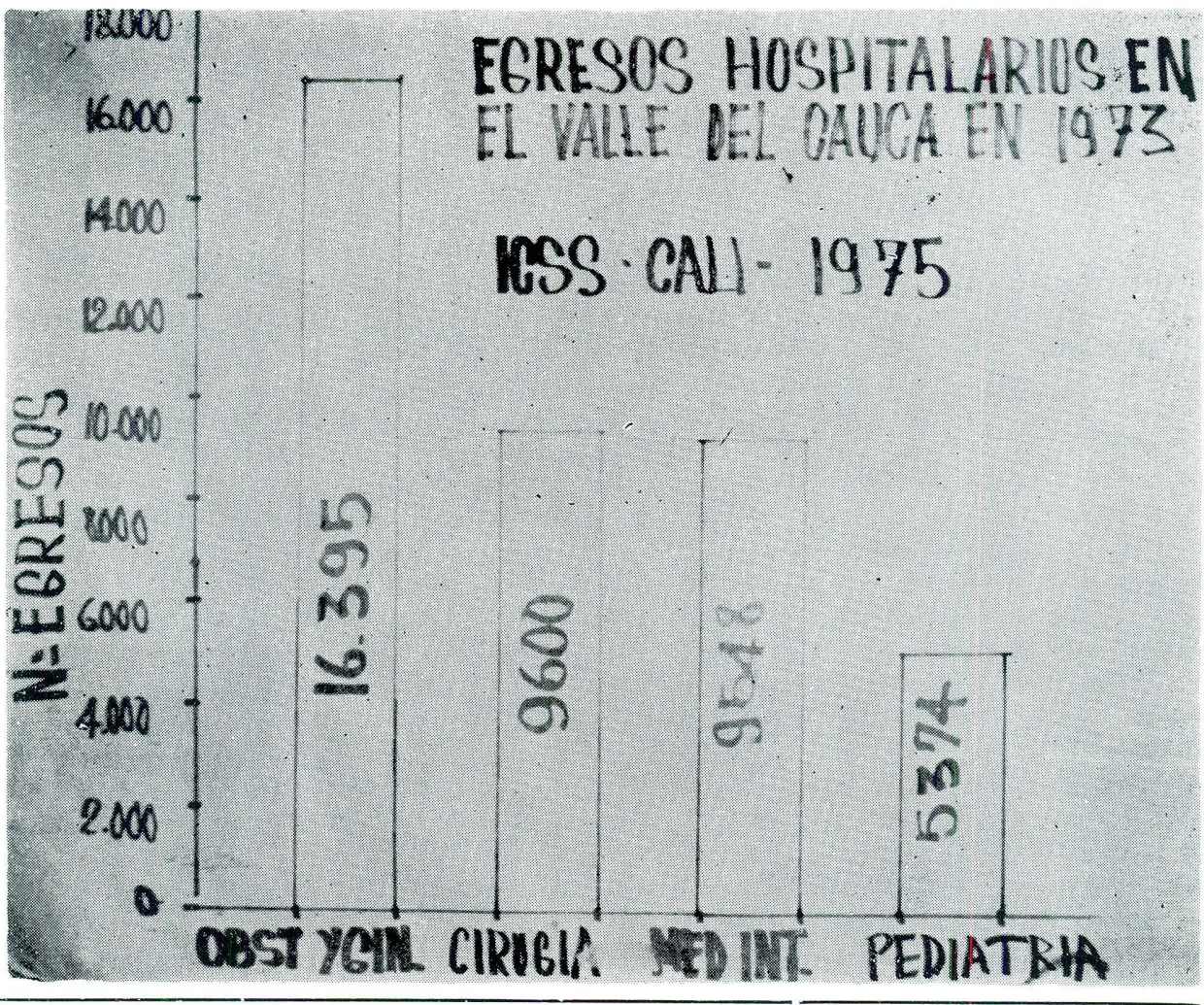

GRAFICA $\quad$ № 6

DISTRIBUCION DE AFILIADOS, PARTOS Y TASAS

DE PARTOS POR MIL AFILIANOS PARA CALI Y

VALLE EN LOS ANOS 1970-19771-1972-1973-1974

\begin{tabular}{|c|c|c|c|c|c|c|}
\hline & & 170 & & 1972 & 1973 & 1974 \\
\hline & VALIE & 22 & $\begin{array}{r}7.373 \\
12.657\end{array}$ & 11.626 & $\begin{array}{l}6.921 \\
11.465\end{array}$ & \\
\hline & $\begin{array}{l}\text { CALI } \\
\text { VALLI }\end{array}$ & $\begin{array}{l}10.721 \\
180.167\end{array}$ & $\begin{array}{l}108.566 \\
178.039\end{array}$ & $\begin{array}{l}119.353 \\
199.000\end{array}$ & $\begin{array}{l}129.900 \\
213.10\end{array}$ & 211.74 \\
\hline & $\begin{array}{l}\text { CAL } \\
\text { VAI }\end{array}$ & 70.7 & 70.0 & $\begin{array}{l}58.8 \\
58.4 \\
\end{array}$ & $\begin{array}{l}53.3 \\
53.8 \\
\end{array}$ & $\begin{array}{l}52.2 \\
50.4\end{array}$ \\
\hline
\end{tabular}




$$
M
$$


GRA:FICA № 9

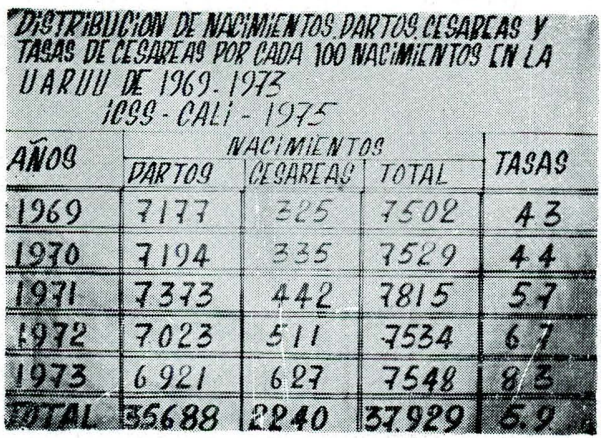

Se estudia la tasa de abortos por 1000 embarazos en pacientes afiliadas y berieficiarias según condición y estado civil. (CUADRO N ? 4).

Se hace resumen gráfico de la campaña de Profamilia durante los años 1973 y 1974 mostrando el número de inserciones inmediatas post partos y mediatas en Profamilia y el número de pacientes motivadas (GRAFICA $\mathrm{N} \div 11$ ).

\section{CUADRO NN 3}

Tasa de Embarazo por 1.000 entre Afiliadas y Bereficiarias

ICSS — Valle de 1967 a 1974

A F I L I A D A

\begin{tabular}{lrrrrrr} 
ANO & N & $\begin{array}{c}\mathrm{N}^{\circ} \\
\text { Emb. }\end{array}$ & TASA & $\mathrm{N}^{\circ}$ & $\begin{array}{c}\mathrm{N}^{\circ} \\
\text { Emb. }\end{array}$ & TASA \\
\hline 1967 & 31618 & 1930 & $\mathbf{6 2 . 2}$ & 45232 & 11524 & $\mathbf{2 5 4 . 7}$ \\
1968 & 30937 & 2280 & $\mathbf{7 3 . 7}$ & 49090 & 11816 & $\mathbf{2 4 0 . 7}$ \\
1969 & 34314 & 2468 & $\mathbf{7 1 . 9}$ & 61273 & 11809 & $\mathbf{1 9 2 . 7}$ \\
1970 & 38716 & 2667 & $\mathbf{6 8 . 9}$ & 62374 & 12019 & $\mathbf{1 9 2 . 7}$ \\
1971 & 39883 & 3031 & $\mathbf{7 6 . 5}$ & 63524 & 11695 & $\mathbf{1 8 4 . 3}$ \\
1972 & 47780 & 3464 & $\mathbf{7 2 . 5}$ & 68912 & 10378 & $\mathbf{1 5 0 . 6}$ \\
1973 & 53769 & 3791 & $\mathbf{7 0 . 5}$ & 72094 & 9846 & $\mathbf{1 3 6 . 5}$ \\
1974 & 57661 & 4120 & $\mathbf{7 1 . 4}$ & 75177 & 9340 & $\mathbf{1 2 4 . 2}$
\end{tabular}




\section{U A D R O No 4}

Tasa de Abortos por 1.000 Embarazos según condición y Estado Civil entre Afiliadas y Beneficiarias del ICSS - Valle 1971

\begin{tabular}{|c|c|c|c|c|c|c|c|c|c|}
\hline \multirow[b]{2}{*}{ ESTADO } & \multicolumn{3}{|c|}{ AFILIADA } & \multicolumn{3}{|c|}{ BENEFICIARIA } & \multicolumn{3}{|c|}{$\mathrm{T} O \mathrm{~T} A \mathrm{~L}$} \\
\hline & $\begin{array}{l}\mathrm{N}^{\circ} \\
\text { Emb. }\end{array}$ & $\begin{array}{c}\mathrm{N}^{\prime \prime} \\
\text { Aborto }\end{array}$ & TASA & $\begin{array}{c}\mathrm{N}^{\circ} \\
\text { Emb. }\end{array}$ & $\begin{array}{c}\text { No } \\
\text { Aborto }\end{array}$ & TASA & $\begin{array}{c}N^{\circ} \\
\text { Aborto }\end{array}$ & $\begin{array}{c}\mathrm{N}^{\circ} \\
\text { Emb. }\end{array}$ & TASA \\
\hline SOLTERA & 1120 & 391 & 349.1 & 459 & 51 & 111.1 & 1579 & 442 & 279.0 \\
\hline CASADA & 1633 & 339 & 207.6 & 10390 & 1247 & 117.7 & 12223 & 1586 & 129.6 \\
\hline VIUDAD & 15 & 10 & 666.7 & 14 & - & - & 29 & 10 & 548.8 \\
\hline UNION LIBRE & 286 & 60 & 209.8 & 638 & 90 & 141.7 & 921 & 130 & 162.7 \\
\hline TOTAL & 3055 & 800 & 262.2 & 11693 & 1388 & 118.7 & 14753 & 2188 & 148.4 \\
\hline
\end{tabular}




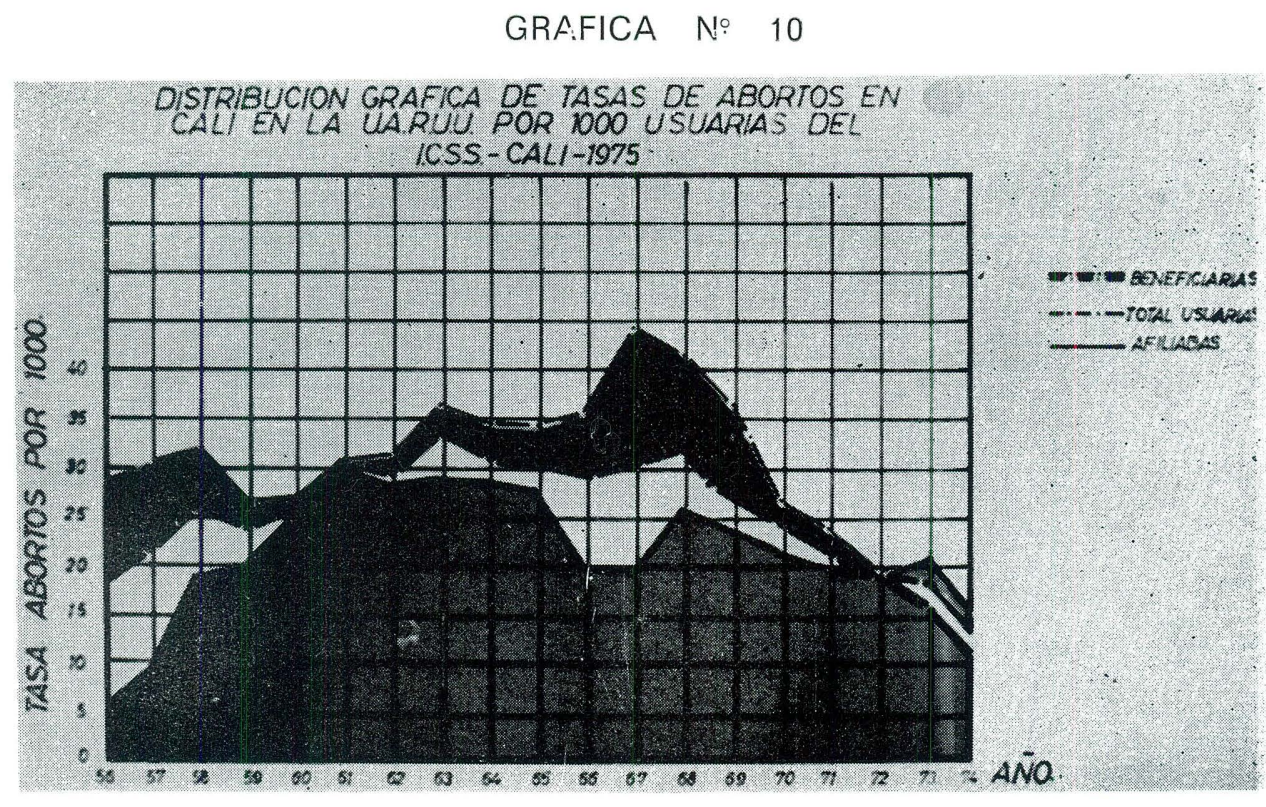

\section{GRAFICA N: 11}

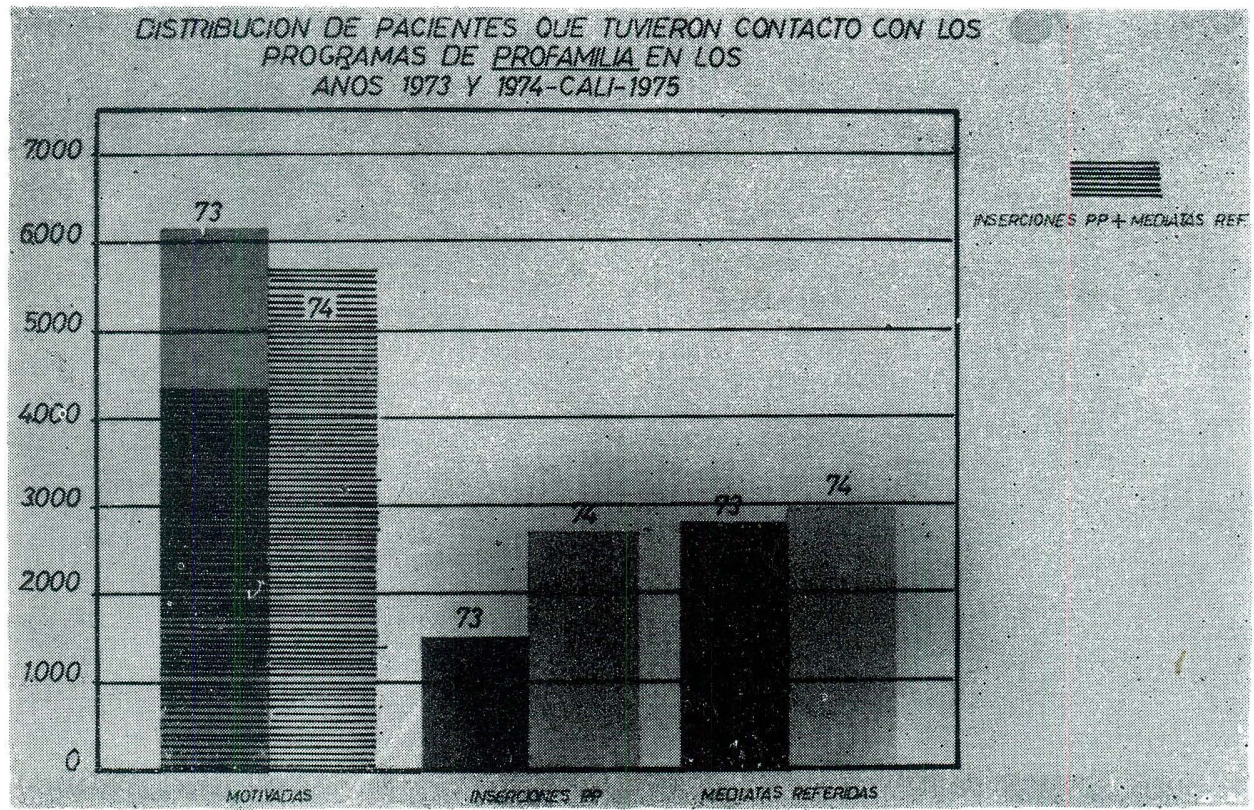




\section{Resultados}

El $40 \%$ de las causas de hospitalización en el ICSS corresponden a pacientes obstétricas. En una población, con condiciones socioEconómicas más o menos iguales, se encuentra una definida diferencia en tasas de nacimientos.

Fue posible obtener una franca disminución de las tasas de nacimientos en el Valle y Cali, aún con políticas tímidas de planificación Familiar.

Se señalan las tasas de embarazos entre afiliadas y beneficiarias del año 1956 a 1974 encontrando una franca disminución a partir del año 1969 que se organizó Profamilia y una gran diferencia entre afiliadas y beneficiarias con una caída a expensas de las tasas de nacimientos de las beneficiarias.

Es de suponer que el grupo de pacientes afiliadas está más expuesto a promiscuidad, tienen protección y halagos con la incapacidad de 56 días y es posible que una gran parte han sido afiliadas únicamente para la atención del parto.

El aumento en los porcentajes de cesáreas puede explicarse con unas técnicas más depuradas en cuanto a salud del niño y en algún porcentaje a procedimientos que se hacían anteriormente como medio para practicar la ligadura de trompas.

El número de abortos ha disminuido considerablemente en las pacientes beneficiarias y muy poco o casi estable en afiliadas. Podríamos pensar que las afiliadas que tienen menos acceso a las campa- ñas de planificación, que muchos de estos abortos pueden ser provocados pues son pacientes en condiciones socio-económicas diferentes a las beneficiarias. Además el estado civil de estas pacientes, una alta tasa de solteras, es una de las consecuencias lógicas.

\section{Conclusiones}

1) Las campañas de Planificación Familiar han creado una conciencia y han demostrado su resultado en la franca disminución de las tasas de embarazos y abortos.

2) Las diferencias existentes entre las tasas de partos y abortos en los grupos de afiliadas y beneficiarias indica que debe haber una revisión a la reglamentación de la Ley del ICSS en cuanto a incapacidades y otros aspectos en atención de partos y abortos en afilia. das.

3) Se justifica en el ICSS invertir en campañas de Planificación familiar, educación sexual, etc. cuyos resultados serían francamente retribuibles económica y socialmente.

4) Se dan normas en cuanto a Planificación aue han sido ampliamente aceptadas por la Institución, que pueden constituir una solución intermedia en cuanto a política demográfica respecta.

\section{Conclusión:}

1. The Family Planning Campaigns have create a conscience and have shown its results in the frank decrease of the Parturition and Abortion Tasas. 
2. The existing differences between Parturition and Abortion Tasas in the affiliated and beneficed shows that there must be a revisal of the stablishments of rules and regulations of the I.C.S.S.'s Law a bout incapacity and some aspects in attention of Parturition and Abortion in the Affiliated.

3. Is worth in the 1.C.S.S. to invest in campaigns of Family Planning, Sexual Education, etc., which results woulb be a complete economical and so. cial Fee.

4. There are given some rules about Family Planning that have been completly accepted by the Institution that can construct and Interpose Solution about Demographic Politics.
BIBLIOGRAFIA

Cipolla M. C. - LA EXPLOSION DEMOGRAFICA. Salvat V 15.

Cottier G. - REGULACION DE LA NATALIDAD. PROBLEMAS SOCIOLOGICOS Y MORALES, Madrid, 1971.

Hartley F. S. - POPULATION QUANTITY vs. QUANTITY A SOCIOLOGICAL EXAMINATION OF THE CAUSES AND CONSEQUENCES OF THE POPULATION EXPLOSION.

London, 1972.

Sauvy A. - CRECIMIENTO CERO? Barcelona, 1973.

The Population Council. - Colombia. Perspectivas por países. 